\title{
Evaluación del trabajo esencial de fractura en chapa de un acero de alta resistencia de fase dual $\left.\right|^{(\bullet)}$
}

\author{
D. Gutiérrez*, Ll. Pérez*, A. Lara*, D. Casellas*y** y J.M. Prado*y **
}

\begin{abstract}
Resumen
La tenacidad de fractura de aceros de alta resistencia (AHSS), es una propiedad interesante para optimizar el comportamiento a impacto, pero su caracterización experimental en chapas metálicas es compleja debido a su espesor. En este trabajo se plantea el método del Trabajo Esencial de Fractura (TEF), como alternativa al cálculo de la tenacidad. Este método ya ha sido utilizado con éxito en polímeros y aceros dúctiles, aunque la información sobre su aplicación en AHSS es escasa. Se ha evaluado la tenacidad en un acero de fase dual de $800 \mathrm{MPa}$ de resistencia y se ha comparado con la de un acero de embutición. Los resultados muestran que se cumplen los requisitos del método y ponen en evidencia la influencia del radio de entalla en el valor obtenido de TEF, especialmente para AHSS. Con todo ello, el método TEF se puede usar para caracterizar la tenacidad de AHSS en formato chapa.
\end{abstract}

Palabras clave Trabajo esencial de fractura; TEF; AHSS; Tenacidad de fractura; Radio de entalla.

\section{Evaluation of essential work of fracture in a dual phase high strength steel sheet}

Abstract

Keywords

\begin{abstract}
Fracture toughness of advanced high strength steels (AHSS), can be used to optimize crash behavior of structural components. However it cannot be readily measured in metal sheet because of the sheet thickness. In this work, the Essential Work of Fracture (EWF) methodology is proposed to evaluate the fracture toughness of metal sheets. It has been successfully applied in polymers films and some metal sheets. However, their information about the applicability of this methodology to AHSS is relatively scarce. In the present work the fracture toughness of a Dual Phase (strength of $800 \mathrm{MPa}$ ) and drawing steel sheets has been measured by means of the EWF. The results show that the test requirements are met and also show the clear influence of notch radii on the measured values, specially for the AHSS grade. Thus, the EWF is postulated as a methodology to evaluate the fracture toughness in AHSS sheets.
\end{abstract}

Essential work of fracture; EWF; AHSS; Fracture toughness; Notch sharpening.

\section{INTRODUCCIÓN}

En los últimos años, la reducción del peso de los vehículos, sin perjuicio de su comportamiento a impacto, ha experimentado un avance muy importante gracias al desarrollo de los aceros avanzados de alta resistencia (AHSS). Actualmente, estos aceros se usan ampliamente en componentes estructurales de chasis y carrocería, y se caracterizan por combinar elevados valores de resistencia mecánica (desde 600 hasta $1500 \mathrm{MPa}$ ) con aceptables niveles de ductilidad. La figura 1 muestra los actuales aceros AHSS y los nuevos grados en fase de desarrollo, que en un futuro podrían incre- mentar aún más esta combinación de propiedades en comparación con los aceros de conformado en frío convencionales ${ }^{[1]}$.

El comportamiento a tracción y conformabilidad de estos nuevos aceros ha sido objeto de amplios estudios en los últimos años, y hoy en día se tiene un buen conocimiento de su relación microestructurapropiedades mecánicas. Sin embargo, menos atención ha recibido su comportamiento a fractura, en particular su resistencia a impacto o su tenacidad de fractura. Estas propiedades son sumamente interesantes para optimizar el comportamiento a impacto de componentes estructurales en vehículos.

(•) Trabajo recibido el día 15 de febrero de 2012 y aceptado en su forma final el día 6 de junio de 2012.

* Fundació CTM Centre Tecnològic, Av. Bases de Manresa, 1, 08242 Manresa, Spain.

** Department of Materials Science and Engineering Metallurgy, Universitat Politècnica de Catalunya, EPSEM, Av. Bases de Manresa 61, 08242 Manresa, Spain.E-mail: david.gutierrez@ctm.com.es. 


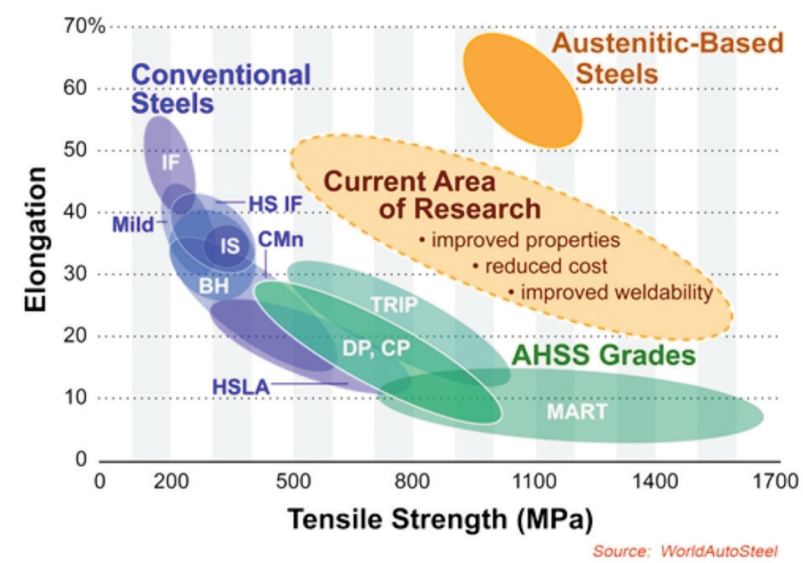

Figura 1. Comparación del alargamiento a fractura con la resistencia a tracción para diferentes aceros de conformado en frío convencionales y de alta resistencia ${ }^{[1]}$.

Figure 1. Comparison of elongation at fracture with tensile strength for different conventional cold forming and high strength steels ${ }^{[1]}$.

La tenacidad de fractura $\left(K_{I C}\right)$ es la medida de la resistencia de un material a la propagación de una grieta, por lo que es una propiedad del material que puede ayudar en el diseño de componentes resistentes a impactos. El conocimiento de esta propiedad para los aceros AHSS permitiría discernir qué aceros de esta familia absorben más energía en un proceso de fractura, y por tanto permitirían optimizar la relación peso-resistencia a impacto en componentes de seguridad sometidos a impactos. Sin embargo, tal como se expone a continuación, la determinación experimental de $K_{I C}$ para chapa metálica presenta dificultades, por lo que no es actualmente un parámetro utilizado en diseño.

En estados de deformación plana, la tenacidad es un parámetro intrínseco del material. Por este motivo, los ensayos estandarizados para medir tenacidad imponen restricciones geométricas en las probetas para asegurar que el estado tensional es de deformación plana $^{[2]}$. Según la norma ASTM E-399, el espesor, $B$, debe ser $B>2,5\left(K_{I C} / \sigma_{0}\right)^{2}$, donde $\sigma_{0}$ es el límite elástico. Para un acero al carbono se reportan valores de $J_{I C}$ y $\sigma_{0}$ de $798 \mathrm{~kJ} / \mathrm{m}^{2}$ y $304 \mathrm{MPa}$ respectivamente ${ }^{[3]}$. Mediante la relación de $K_{I C}=\left(E \cdot J_{I C}\right)^{1 / 2}$, indicaría que $B$ debe ser superior a $4.000 \mathrm{~mm}$. Obviamente, estas condiciones distan de poder cumplirse en productos planos como la chapa metálica, de 1-3 mm de espesor, comúnmente usados en componentes estructurales en automoción. La metodología del Trabajo Esencial de Fractura, TEF (Essential Work of Fracture, EWF) fue desarrollada para llenar este vacío, a partir de la idea de Broberg ${ }^{[4]}$, que se basa en separar la energía de fractura de la energía asociada con la deformación plástica desarrollada delante de la punta de la fisura. En 1977 Cotterell y Reddell[5] definieron el método para chapa metálica. Así, el objetivo de esta metodología es cuantificar la energía por unidad de área consumida en la formación de superficies de fractura. Es en este sentido que se considera un trabajo esencial, siendo por la forma en la que está definido, una manera de aproximar el valor de $J_{c^{*}}$. Esta metodología se ha aplicado anteriormente para caracterizar chapas de acero, aleaciones de aluminio, bronces y latones ${ }^{[6-8]}$. Los buenos resultados conseguidos permiten postular su uso para aceros AHSS como herramienta de caracterización de su tenacidad a fractura.

Puesto que el trabajo esencial de fractura es la energía necesaria para que se fracture el material delante de la punta de una grieta y que ésta avance, la evaluación de esta propiedad requiere introducir entallas muy afiladas en las probetas con el fin de simular el efecto de una grieta real. Esta problemática también se da en la evaluación de la tenacidad de fractura en deformación plana, donde es bien conocido que por debajo de un valor crítico de radio de entalla, la tenacidad de fractura medida es independiente del radio de entalla. Por tanto se puede considerar como una propiedad intrínseca del material $^{[3}$ y 9$]$. Para evitar el efecto del radio de entalla, el procedimiento de la norma ASTM E-399, para evaluar la tenacidad de fractura, sugiere la nucleación de una grieta por fatiga en la entalla. Siguiendo las recomendaciones de esta norma de ensayo, en la evaluación del TEF se debe tener en cuenta el efecto del radio de entalla, utilizando radios tan pequeños como sea posible y valorar, en cada caso, la generación de grietas por fatiga a partir de entallas mecanizadas.

Así pues, el objetivo de este trabajo es valorar la aplicabilidad del método del Trabajo Esencial de Fractura para evaluar la tenacidad de fractura de un acero de fase dual, DP780, comúnmente usado en automoción en formato chapa para componentes con responsabilidad en aspectos de seguridad pasiva, teniendo en cuenta el efecto del radio de entalla en su evaluación. Los resultados obtenidos se comparan con los de un acero de bajo carbono y alta embutibilidad.

\section{DETERMINACIÓN DEL TRABAJO ESENCIAL DE FRACTURA}

Actualmente no existe ninguna normativa que describa la determinación del trabajo esencial de fractura en materiales en forma de láminas, tan sólo existe un protocolo resultado de la experiencia en el uso 
del método de un conjunto de laboratorios, elaborado por la Sociedad Europea de Integridad Estructural (European Structural Integrity Society, ESIS) ${ }^{[10]}$. Sin embargo su uso está muy extendido, particularmente, en la determinación de la tenacidad en films poliméricos $^{[11-13]}$. En metales, ha sido utilizado para caracterizar diferentes chapas metálicas de aleaciones de zinc $^{[6]}$, aleaciones de aluminio ${ }^{[14]}$, bronce y latón ${ }^{[8]}$, aceros de baja aleación ${ }^{[5}$ y 7$]$ y, recientemente, en aceros TRIP[15].

La metodología desarrollada por Cotterell y Reddel en $1977^{[5]}$ propone que el trabajo necesario para hacer avanzar una grieta en un material dúctil se puede separar en dos contribuciones: la energía correspondiente al trabajo de deformación plástica en el frente de la grieta y la energía consumida por todos los procesos microscópicos que causan la fractura. Estas dos energías se consumen en dos zonas diferenciadas en el frente de la grieta. Por un lado, delante de la punta de la grieta se puede definir una zona plástica, en la que el nivel de esfuerzos conduce a la fluencia plástica del material, sobre la que se superpone una zona muy pequeña, situada justo delante del frente de avance de la grieta, llamada zona de proceso (Fracture Process Zone - FPZ, (Fig. 2 a)) en la que se producen los procesos propios de la fractura.

Por tanto, es posible separar la energía de fractura, $W_{f}$, en dos componentes: $W_{p}$, la energía consumida en la zona plástica, y $W_{\mathrm{e}}$, la energía consumida en la zona de proceso. Por tanto:

$$
W_{f}=W_{e}+W_{p}
$$

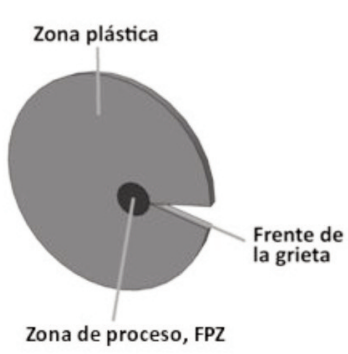

(a)

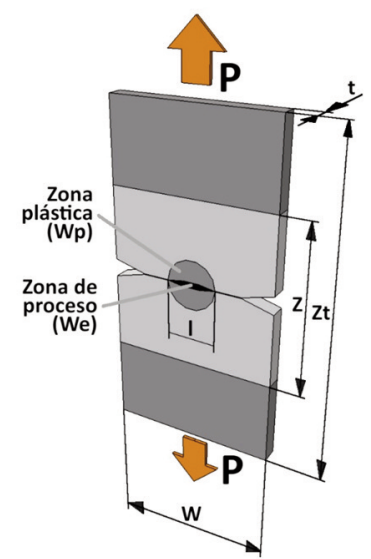

(b)
Figura 2. (a) Zona plástica y de proceso, FPZ, delante de la punta de la fisura. (b) Probeta doblemente entallada, tipo DENT.

Figure 2 (a). Plastic and process zone, FPZ, in front of the crack tip. (b) Double edge-notched sample, DENT.
En general, la ecuación (1) suele operarse siempre con los trabajos por unidad de superficie fracturada, $w_{f}, w_{p}$ y $w_{e}$. En este caso, la geometría y el trabajo consumido en la zona plástica dependen de la geometría y el modo de carga, motivo por el que $w_{p}$ no puede considerarse una propiedad del material. En cambio $w_{\mathrm{e}}$ sí que es una propiedad del material y se conoce como trabajo esencial de fractura, pudiéndose considerar como la energía necesaria para el avance de una grieta una vez que el material ha desarrollado toda su capacidad de deformación plástica.

Siendo estrictos, el trabajo esencial no es una propiedad intrínseca del material. En función del nivel de constreñimiento en la zona de proceso se disipará más o menos energía para hacer avanzar la grieta. En productos planos, como la triaxialidad de los esfuerzos es mucho menor, se produce estricción delante de la grieta. Así, en la energía consumida en la zona de proceso se tiene en cuenta la energía plástica necesaria para formar la estricción y ésta depende del espesor de la chapa ${ }^{[8]}$. Por tanto, en realidad el valor de $w_{e}$ es una propiedad del material para un determinado espesor.

La evaluación del TEF se realiza en probetas entalladas. Si el material delante de la punta de la entalla o fisura está completamente plastificado y la zona plástica está confinada entre las dos entallas, $W_{p}$ es proporcional al volumen y $W_{\text {e }}$ es proporcional al área fracturada. Así $W_{p}$ y $W_{\mathrm{e}}$ se escalan de forma diferente con el tamaño de la muestra. De esta manera si se ensayan probetas geométricamente similares de distinto tamaño, los dos trabajos de fractura se pueden separar. En principio cualquier geometría de probeta es válida para este ensayo, pero para chapas delgadas las probetas tipo DENT (Double Edge Notched Tension) son especialmente interesantes puesto que los esfuerzos transversales entre las entallas son de tracción y no se generan componentes de flexión o compresión. Para esta geometría de probeta y tipo de carga se observa que, en condiciones de tensión plana, si el ligamento $l$ se encuentra completamente plastificado antes de iniciarse la fractura, la zona plástica creada tiene una geometría circular con diámetro igual a la longitud del ligamento. Durante la fractura dúctil, la energía total absorbida, se puede separar en dos componentes: i) el trabajo esencial de fractura $\left(w_{\mathrm{e}}\right)$ consumido en la FPZ, y ii) el trabajo plástico no-esencial $\left(w_{p}\right)$ disipado en una región externa que envuelve a la FPZ:

$$
W_{f}=w_{e}\left|t+w_{p} \beta t\right|^{2}
$$


donde $\beta$ es un factor de forma que depende de la geometría de la zona plástica, $t$ es el espesor y $l$ es la longitud del ligamento entre las dos entallas. El trabajo específico de fractura $w_{f}$ se obtiene dividiendo la ecuación (2) por el área del ligamento inicial $(l t)$. Por lo tanto, $w_{f}$ se puede expresar como:

$$
\frac{W_{f}}{\text { It }}=w_{f}=w_{e}+w_{p} \beta I
$$

Si $w_{f}$ se grafica en función de la longitud del ligamento $l$, la línea recta que intercepta el valor de ordenada en el origen proporciona el valor de $w_{\text {e }}$. Sin embargo, hay algunas restricciones que se deben tener en cuenta para utilizar la ecuación (3): el área del ligamento debe estar completamente plastificada antes de que se inicie la fractura y el ligamento debe estar en un estado de tensión plana. Para cumplir con estas restricciones y de acuerdo con el protocolo de la ESIS para la determinación del valor $w_{e}$, la longitud menor del ligamento debe ser de 3 a 5 veces el espesor de la chapa, $\left(3 t<1_{\min }<5 t\right)^{[5]}$. La longitud mayor del ligamento no debe ser más grande que $1 / 3$ de la anchura de la muestra $(W / 3)$ o 2 veces el radio de la zona plástica $\left(r_{p}\right)$ en estado de tensión plana en la punta de la grieta ${ }^{[6}$ y 7$]$. De acuerdo con la ecuación (4), $r_{p}$ se define como:

$$
r_{p}=\frac{1}{2 \pi}\left(\frac{K}{\sigma_{y}}\right),
$$

donde $K$ es el factor de intensidad de tensiones y $\sigma_{y}$ es el límite elástico del material. En estas condíciones, el valor $w_{e}$ puede ser considerado como la resistencia a la propagación de una grieta en condiciones de estado estacionario, así como el valor crítico de la integral-J en el inicio de la grieta, $J_{c}{ }^{[12]}$.

\section{MATERIALES Y MÉTODO EXPERIMENTAL}

Se han caracterizado dos aceros en formato de chapa con 1,5 mm de espesor; un acero de fase dual con una resistencia a tracción de $780 \mathrm{MPa}$, conocido comúnmente como DP780, y un acero de bajo carbono y alta embutibilidad denominado como DC03. Las propiedades mecánicas y composición química de los aceros estudiados se resumen en la tabla I, mientras que la figura 3 muestra la microestructura de los materiales analizados.

Las microestructuras se han revelado mediante ataque químico con Nital 2 \%. En el caso del DP780, (Fig. 3 a)), muestra una matriz martensítica con ferrita en borde de grano. Para el acero DC03, (Fig. 3 b)), la microestructura muestra los granos de ferrita de color blanco (alargados en sentido de laminación) con colonias de perlita en color negro.

Las probetas DENT, (Fig. 2 b)), se han extraído en dirección transversal respecto a la dirección de laminación de la chapa, siendo las dimensiones de $240 \mathrm{~mm}$ de longitud y $55 \mathrm{~mm}$ de anchura. Las entallas de estas probetas se han mecanizado por corte por hilo e introducido 2 radios de entalla $(\rho)$ con el fin de evaluar su efecto en el cálculo final del TEF (Fig. 4):

- Radio de $250 \mu \mathrm{m}$ obtenido por corte por hilo.

- Radio inferior a 0,1 $\mu \mathrm{m}$; correspondiente al de una fisura nucleada y propagada por fatiga. Esta se ha obtenido a partir de un radio intermedio, afilando con cuchilla la entalla obtenida por corte por hilo.

El trabajo total de fractura $\left(W_{f}\right)$ se ha calculado mediante ensayo de tracción de las muestras DENT en una máquina de ensayos universal a una velocidad de $1 \mathrm{~mm} / \mathrm{min}$. El desplazamiento se midió mediante videoextensometría con un ancho de calibración de $50 \mathrm{~mm}$. Las probetas DENT ensayadas tenían longitudes de ligamento $(I)$ comprendidas entre 6 y $16 \mathrm{~mm}$ y se ensayaron hasta la rotura de las mismas. Se han ensayado un total de 24 probetas, es decir, 4 probetas

Tabla I. Propiedades mecánicas y composición química de los aceros estudiados

\begin{tabular}{|c|c|c|c|c|c|c|c|c|c|}
\hline \multirow{2}{*}{ Material } & \multirow{2}{*}{$\begin{array}{l}\text { Límite } \\
\text { elástico } \\
\text { [MPa] }\end{array}$} & \multirow{2}{*}{$\begin{array}{c}\text { Resistencia } \\
\text { máxima } \\
\text { [MPa] }\end{array}$} & \multirow{2}{*}{$\mathrm{n}$} & \multirow{2}{*}{$\begin{array}{c}\text { Deformación } \\
\text { a rotura } \\
{[\%]}\end{array}$} & \multicolumn{5}{|c|}{$\begin{array}{l}\text { Composición química } \\
(\% \text { en peso) }\end{array}$} \\
\hline & & & & & C & Si & $M n$ & $\mathbf{P}$ & $\mathrm{Cr}$ \\
\hline DC03 & 186 & 314 & 0,206 & 42 & 0,078 & 0,01 & 0,294 & - & 0,016 \\
\hline DP780 & 559 & 819 & 0,161 & 21 & 0,13 & 0,209 & 1,892 & 0,023 & 0,179 \\
\hline
\end{tabular}

Table I: Mechanical properties and chemical composition of studied steels 


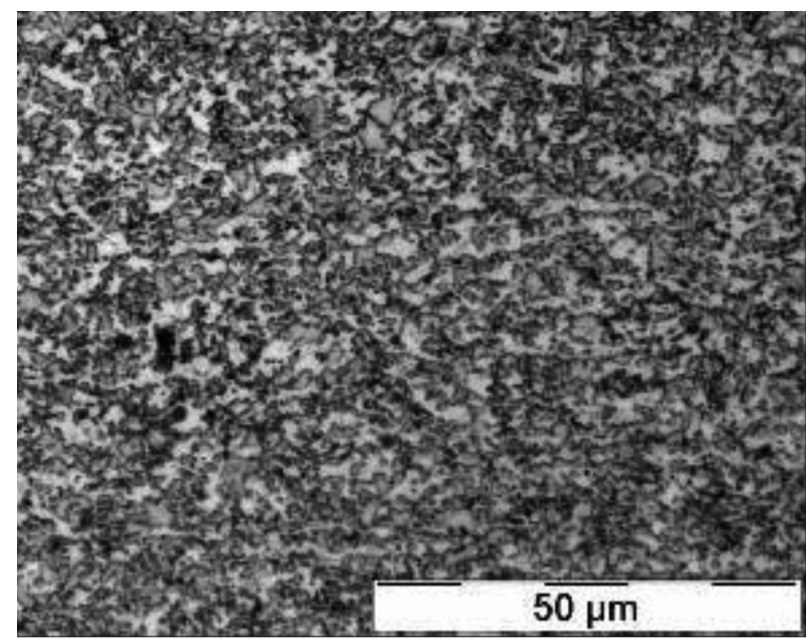

(a)

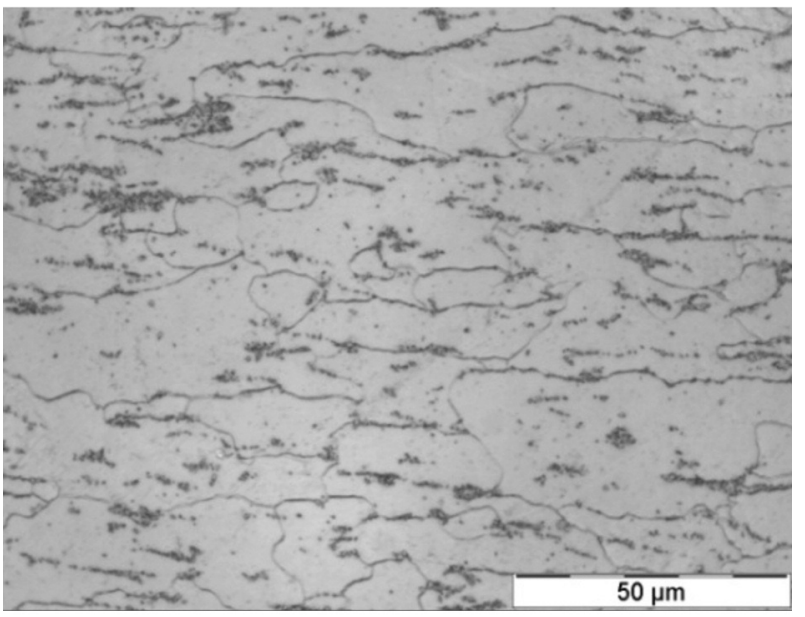

(b)

Figura 3. a) Microestructura del acero DP780. b) Microestructura del acero DC03.

Figure 3. a) Microstructure of DP780 steel. b) Microstructure of DCO3 steel.

por ligamento. El trabajo específico de fractura $\left(w_{f}\right)$ se calculó a partir de la integración de las curvas carga-desplazamiento dividido por el área inicial del ligamento (lt).

El inicio de propagación de la grieta se detectó descargando las muestras de mayor ligamento a diferentes niveles de esfuerzo e inspeccionando, mediante microscopía óptica, el grado de deformación en la punta de la grieta. Además, se utilizó un equipo de medida de deformaciones, GOM-ARAMIS ${ }^{\mathrm{TM}}$ para determinar la deformación en la zona del ligamento durante el ensayo. Este sistema está basado en dos cámaras CCD que permiten detectar la deformación en la superficie de la probeta ${ }^{[16]}$.

Además se han verificado, de acuerdo con el protocolo de la $\mathrm{ESIS}^{[10]}$, las siguientes condiciones:
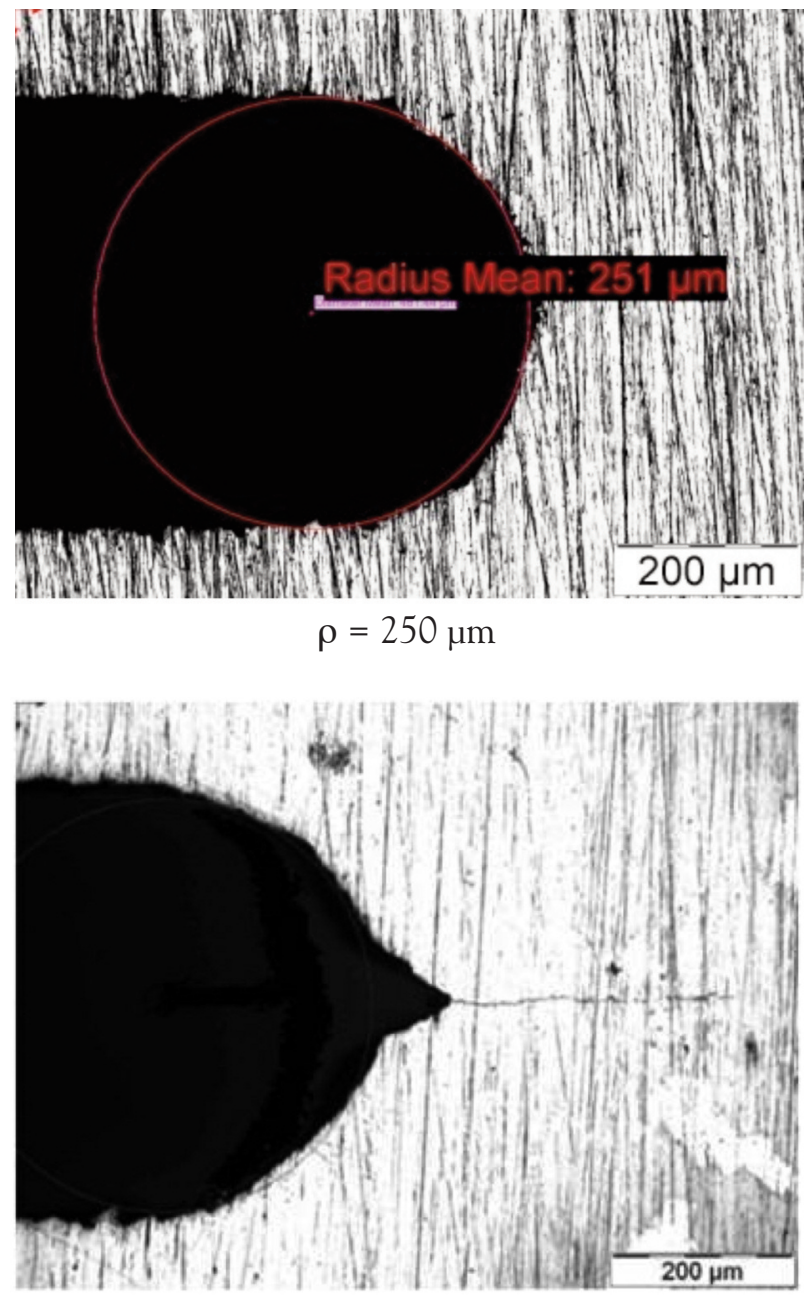

$$
\rho=0,1 \mu \mathrm{m}
$$

Figura 4. Radio de la entalla $(\rho)$ de las probetas DENT.

Figure 4. Notch radii $(\rho)$ of DENT samples.

- Similitud de las curvas carga-desplazamiento, para un conjunto de ligamentos y condiciones de un material.

- Criterio de tensión máxima, donde la máxima tensión alcanzada durante el ensayo $\left(\sigma_{\text {máx }}\right)$ debe estar comprendida entre $1,1 \sigma_{m}<\sigma_{\text {máx }}<0,9 \sigma_{m}$, siendo $\sigma_{m}$ el valor medio de las tensiones máximas.

\section{RESULTADOS}

El método TEF impone que el área del ligamento debe estar totalmente plastificado antes de que la grieta propague, con el fin de asegurar que toda la energía se invierte en crear nuevas superficies y permitir avanzar la grieta y que no se consume en parte en crear deformación plástica. En la figura 5, se mues- 


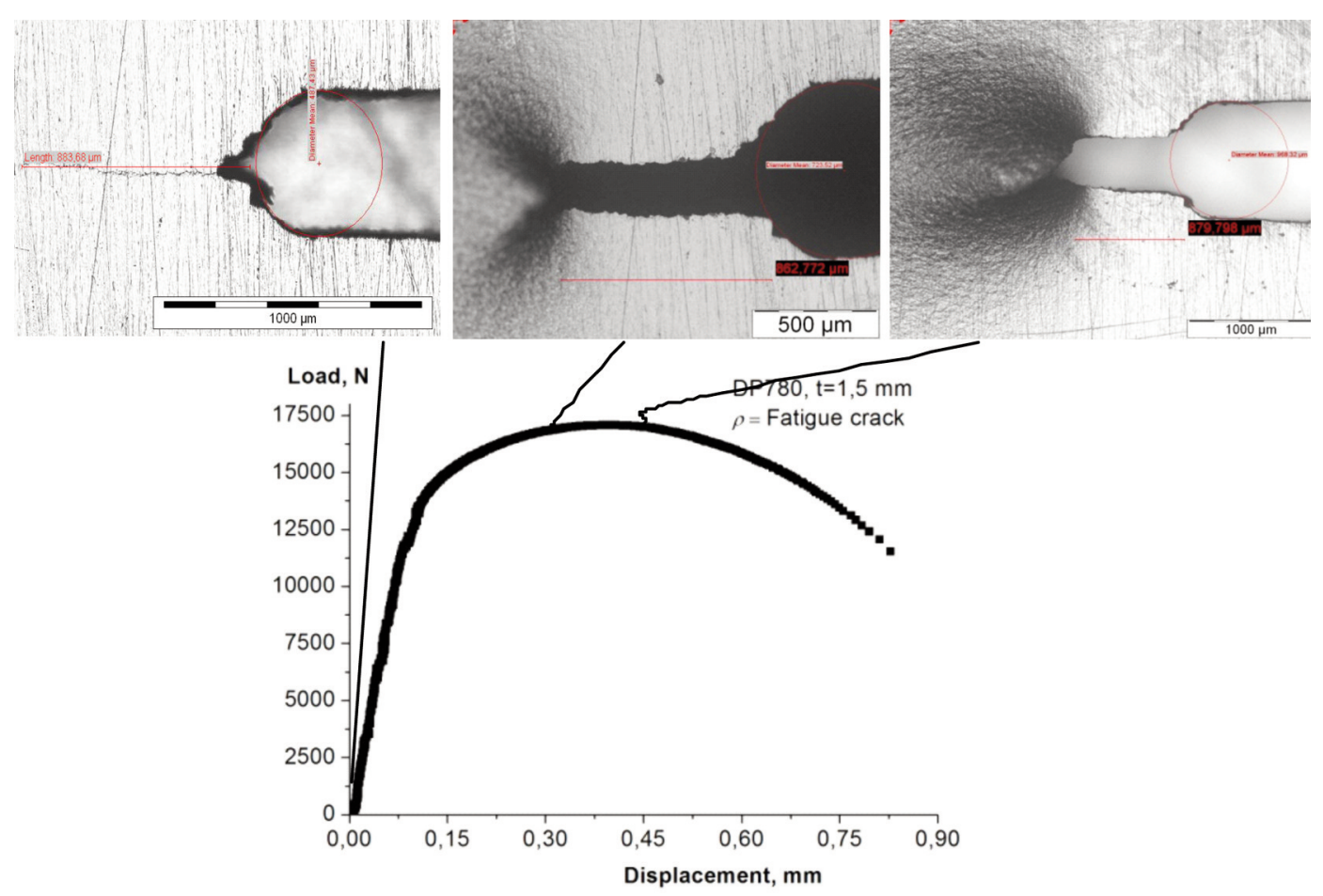

Figura 5. Enromamiento en la punta de la grieta a diferentes estados de carga en acero DP780 con grieta de fatiga inicial.

Figure 5. Blunting at the crack tip at different stages of the test for DP780 with fatigue pre-crack.

tra la punta de la grieta a diferentes estados durante la carga de la probeta.

La punta de la grieta se enroma progresivamente y se observa una zona plástica bien desarrollada cuando se alcanza la carga máxima.

El sistema de medida de deformaciones, ARAMIS, muestra en el DP780 que en carga máxima, con $\mathrm{P}=P_{\max }$, el área del ligamento está completamente plastificado, (Figs. 6 y 7). En estas figuras se muestra el nivel de deformación entre las entallas en el punto de carga máxima para distintos radios de entalla.

Se confirma que la zona plástica está bien desarrollada en la punta de las dos entallas y homogéneamente distribuida entre ellas. Ambos resultados (Figs. 5 y 6) indican que la condición de total plasticidad en los aceros estudiados se cumple y que los valores de $w_{\mathrm{e}}$ pueden ser considerados como válidos.

Así mismo, también se ha verificado la forma de las curvas carga-desplazamiento de los aceros estudiados, (Fig. 8 (radio de entalla de $250 \mu \mathrm{m}$ )), como el criterio de tensión máxima, (Fig. 9 (radio de entalla de $0,1 \mu \mathrm{m})$ ).

Se observa que ambos criterios se cumplen en los dos aceros estudiados y aunque a modo de ejemplo sólo se ha graficado un radio de entalla, ambos criterios se cumplen para los radios de entalla de 250 y
0,1 $\mu \mathrm{m}$. En el caso del DP780, (Fig. 8 b)), alguna de las curvas carga-desplazamiento presenta alguna disimilitud con el resto, pero se ha comprobado que al realizar la regresión lineal por dos veces, tal y como establece el método de la ESIS, estas curvas son descartadas.

La figura 10 muestra los valores de $w_{f}$ con diferente longitud de ligamento $(I)$ y el valor de ordenada en el origen, $w_{\mathrm{e}}$, para los diferentes radios de entalla.

En la figura 11 se comparan los diferentes valores de $w_{e}$ encontrados para los dos aceros estudiados con diferente radio de entalla.

Como se había comentado anteriormente, el procedimiento de la norma ASTM E-399, para evaluar la tenacidad de fractura, sugiere la nucleación de una grieta por fatiga en la entalla; en este caso se han estudiado radios de entalla generados por corte por hilo y por fatiga, analizando su efecto en el valor de $w_{\mathrm{e}}$. La diferencia de valores encontrados, en función del radio de entalla, para el DP780, sugiere que este acero es mucho más sensible a este efecto que el DC03, ya que en este último las variaciones son menores.

En la tabla II se resumen los valores de $w_{\mathrm{e}} \mathrm{y}$ del trabajo no-esencial de fractura (trabajo plástico) para los dos aceros estudiados. 
EVALUACIÓN DEL TRABAJO ESENCIAL DE FRACTURA EN CHAPA DE UN ACERO DE ALTA RESISTENCIA DE FASE DUAL EVALUATION OF ESSENTIAL WORK OF FRACTURE IN A DUAL PHASE HIGH STRENGTH STEEL SHEET

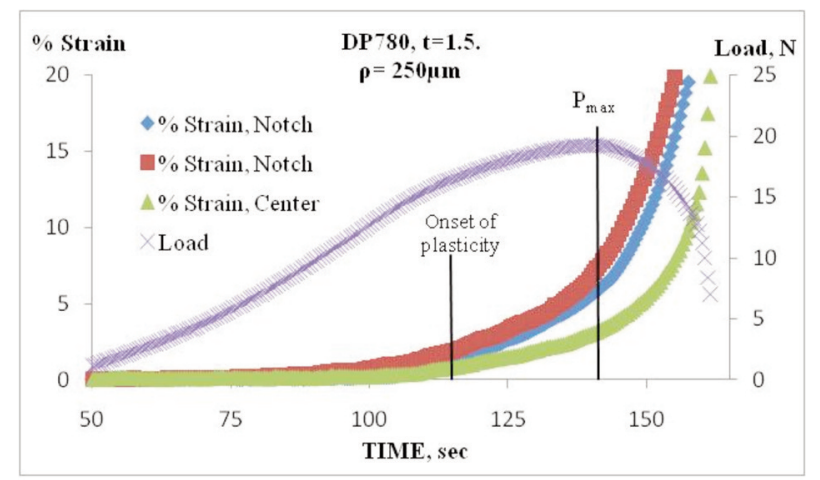

(a)

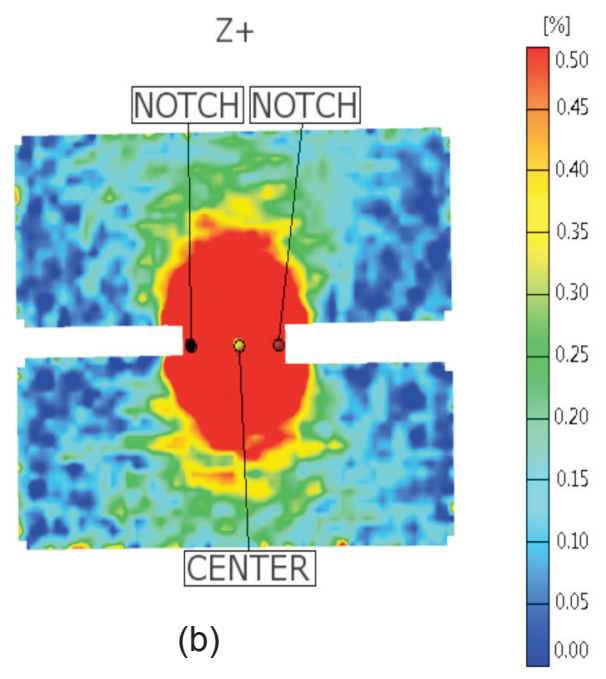

Figura 6. (a) Análisis de la deformación en DP780 con $\rho=250 \mu \mathrm{m}$ durante el ensayo. b) En $\mathrm{P}_{\max }$ el área del ligamento está completamente plastificado.

Figure 6. (a) Strain analysis in DP780 with $\rho=250 \mu \mathrm{m}$ during fracture test. b) At $P_{\max }$ ligament area is fully yielded.

De acuerdo con los valores $w_{\mathrm{e}}$ de la tabla II, se observa una mayor dispersión de resultados en las muestras propagadas por fatiga. Esto es consecuencia

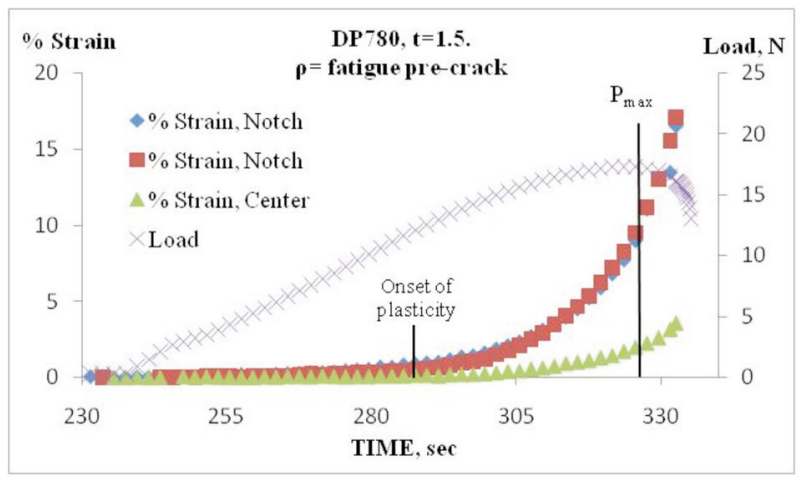

(a)

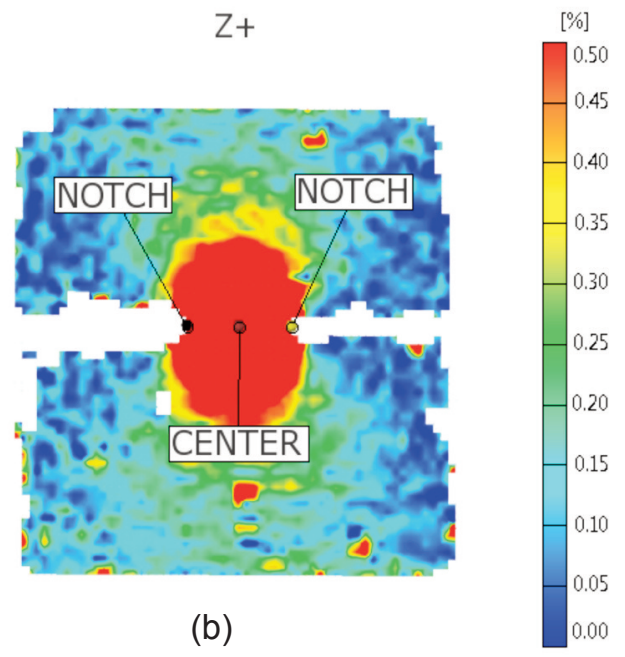

Figura 7. (a) Análisis de la deformación en DP780 con $\rho=0,1 \mu \mathrm{m}$ durante el ensayo. (b) En $\mathrm{P}_{\max }$ el área del ligamento está completamente plastificado.

Figure 7.( a) Strain analysis in DP780 with $\rho=0.1 \mu m$ during fracture test. b) At $P_{\max }$ ligament area is fully yielded.

de las dificultades añadidas al ensayo, ya que se pueden generar grietas de distinto tamaño, más de una grieta por entalla, etc.

Tabla II. Trabajo esencial de fractura $\left(w_{e}\right)$ en los aceros DP780 y DC03

Table II. Essential work of fracture $\left(w_{e}\right)$ of DP780 and DC03 steels

\begin{tabular}{lcccl}
\hline Material & Radio entalla, $\rho(\boldsymbol{\mu m})$ & $\mathbf{w}_{\mathbf{e}}\left(\mathbf{k J / \mathbf { m } ^ { 2 } )}\right.$ & $\mathbf{w}_{\mathbf{p}}\left(\mathbf{k J} / \mathbf{m}^{\mathbf{3}}\right)$ & $\mathbf{R}^{\mathbf{2}}$ \\
\hline DC03 & 250 & $342 \pm 6$ & 46.681 & 0,996 \\
DC03 & 0,1 & $309 \pm 10$ & 44.303 & 0,98 \\
DP780 & 250 & $293 \pm 9$ & 27.061 & 0,98 \\
DP780 & 0,1 & $116 \pm 18$ & 28.754 & 0,96 \\
\hline
\end{tabular}




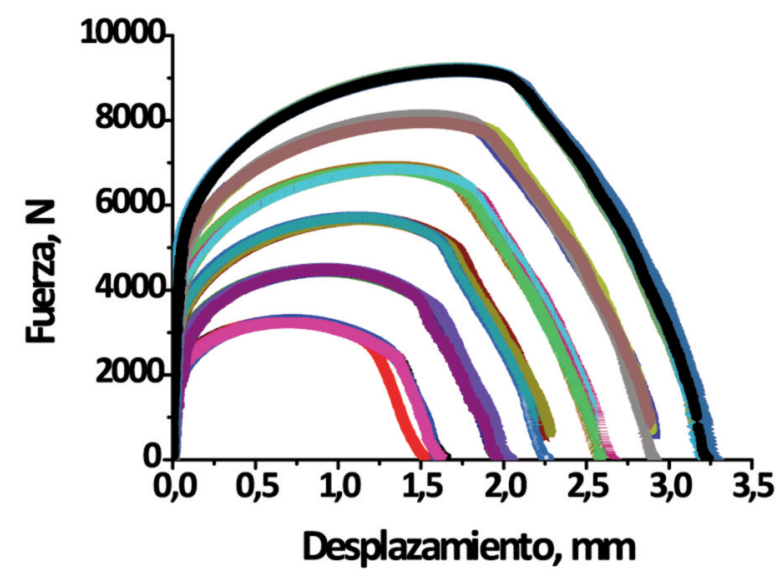

(a)

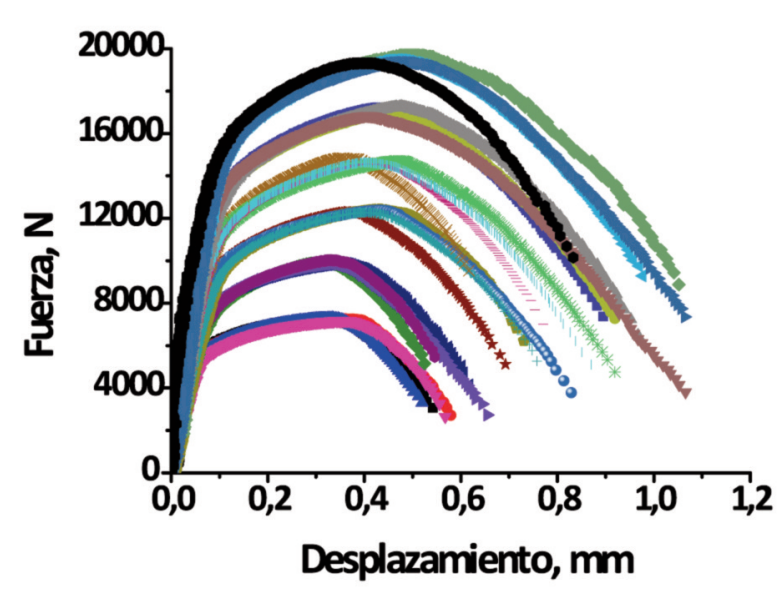

(b)

Figura 8. (a) Curvas carga-desplazamiento para DC03 con $\rho=250 \mu \mathrm{m}$. (b) Curvas cargadesplazamiento para DP780 con $\rho=250 \mu \mathrm{m}$.

Figure 8. (a) Load-displacement diagrams of DC03 with $\rho=250 \mu \mathrm{m}$. (b) Load-displacement diagrams of DP780 with $\rho=250 \mu \mathrm{m}$.

\section{DISCUSIÓN}

La validez del método TEF está basada en la hipótesis de que el área del ligamento debe estar completamente plastificado antes del inicio de propagación de la grieta, es decir, al alcanzar el valor de carga máxima $\left(P_{\text {máx }}\right)$. Mediante el sistema de medida de deformaciones GOM-ARAMIS ${ }^{\text {TM }}$ se ha comprobado experimentalmente que dicha condición se cumple para los aceros estudiados DP780 y DC03. En materiales de elevada ductilidad el método TEF se había aplicado, anteriormente, con excelentes resultados. Para el caso de los analizados, ambos cumplen con las condiciones de plasticidad, por lo que se puede pensar que el método TEF es aplicable tanto en aceros de bajo carbono como en aceros AHSS con resistencias de hasta $800 \mathrm{MPa}$.

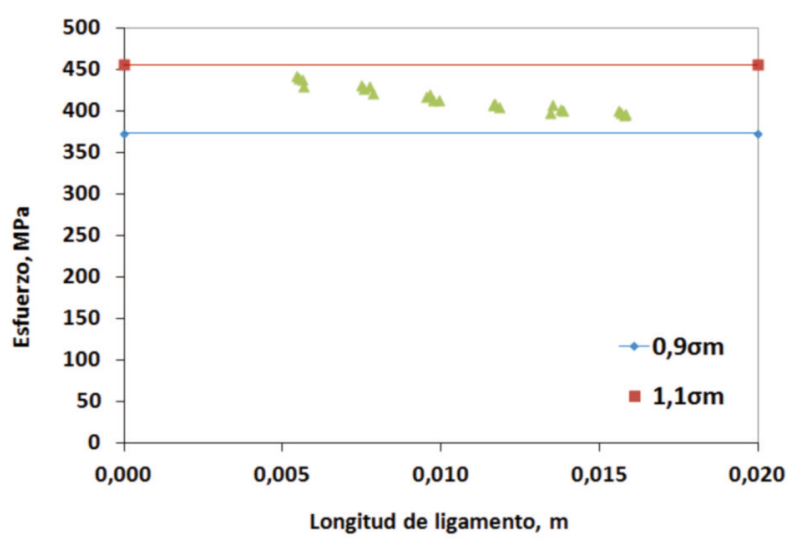

(a)

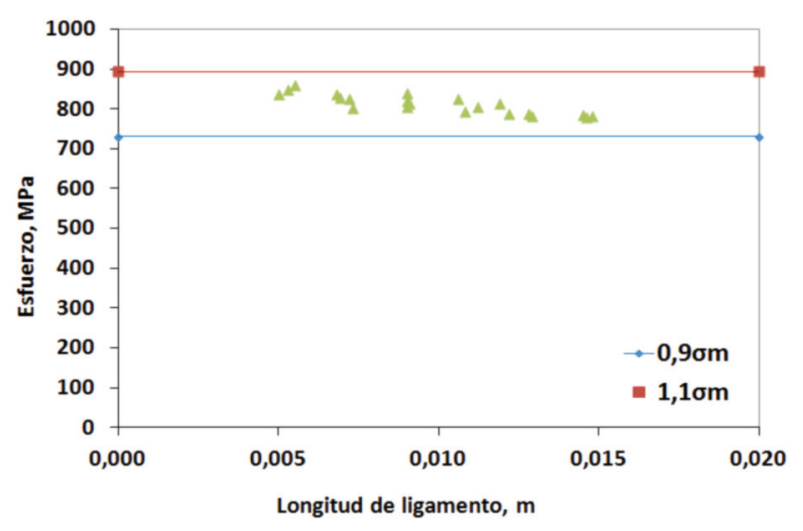

(b)

Figura 9. (a) Criterio de tensión máxima DC03 con $\rho=0,1 \mu \mathrm{m}$. b) Criterio de tensión máxima para DP780 con $\rho=0,1 \mu \mathrm{m}$.

Figure 9. (a) Maximum stress criterion for $\mathrm{DCO} 3$ with $\rho=0.1 \mu \mathrm{m}$. b) Maximum stress criterion for DP780 with $\rho=0.1 \mu \mathrm{m}$.

El valor de $w_{\mathrm{e}}$ en el acero DC03, con $\rho=250 \mu \mathrm{m}$, es de $342 \pm 5,7 \mathrm{~kJ} / \mathrm{m}^{2}$, similar a los valores reportados en bibliografía para aceros de bajo carbono. Knockaert et al. obtuvieron un valor de $400 \pm 15 \mathrm{~kJ} / \mathrm{m}^{2}$ para un acero de embutición de $1,5 \mathrm{~mm}$ de espesor y $225 \mathrm{MPa}$ de límite elástico, con $\rho=200 \mu \mathrm{m}{ }^{[17]}$.

Por otro lado, los resultados obtenidos, para el DP780, del trabajo esencial de fractura, $w_{e}$, muestran una marcada dependencia con el radio de entalla. Los valores de $w_{\mathrm{e}}$ obtenidos con un radio de entalla a partir de una grieta de fatiga son claramente inferiores, $116 \mathrm{~kJ} / \mathrm{m}^{2}$, a los encontrados con radios obtenidos por corte por hilo, $293 \mathrm{~kJ} / \mathrm{m}^{2}$. Para el acero DC03 no se observa esta gran dependencia en los radios de entalla estudiados, los resultados sólo discrepan en un $10 \%$. Para un acero de bajo carbono con límite elástico de $330 \mathrm{MPa}$, parecido al DC03, se ha encontrado que el radio crítico es de $150 \mu \mathrm{m}$ para ensayos de tenacidad con $\mathrm{CTOD}^{[18]}$. Estos resul- 


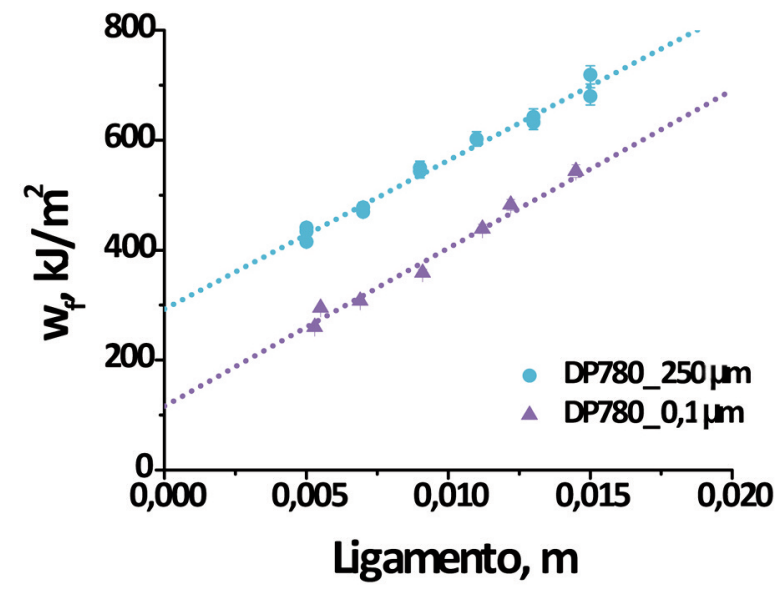

(a)

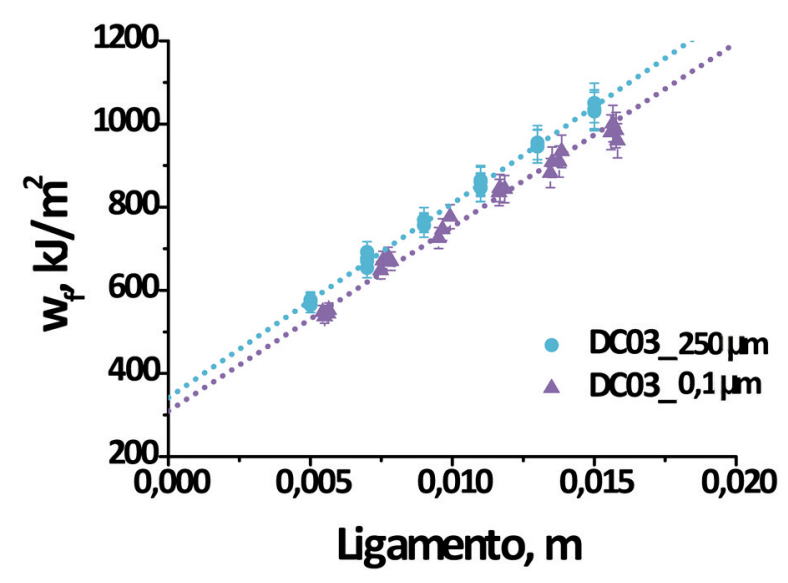

(b)

Figura 10. (a) Valores de $\mathrm{w}_{\mathrm{f}}$ en función del ligamento para DP780. (b) Valores de $w_{f}$ en función del ligamento para DC03.

Figure 10. (a) $w_{f}$ values depending on ligament length of DP780. (b) $w_{f}$ values depending on ligament length of $D C 03$.

tados estarían de acuerdo con la poca dependencia con el radio de entalla determinada experimentalmente para el DC03.

Si se comparan las curvas fuerza-desplazamiento para distintos radios de entalla y un ligamento de $15 \mathrm{~mm}$, se observa la diferencia entre el acero DP780 y el DC03, (Fig. 12).

Estas curvas son muy parecidas en el acero DC03, donde la energía debida a la deformación plástica alrededor de una grieta y previa a la nucleación de una grieta, es independiente del radio de entalla y mucho mayor que la energía necesaria para el crecimiento de la misma. En cambio, en el acero DP780, el radio de entalla condiciona la energía de deformación plástica, siendo ésta superior para entallas menos afiladas, con radio mayor.

Al igual que en los ensayos de tenacidad de frac-

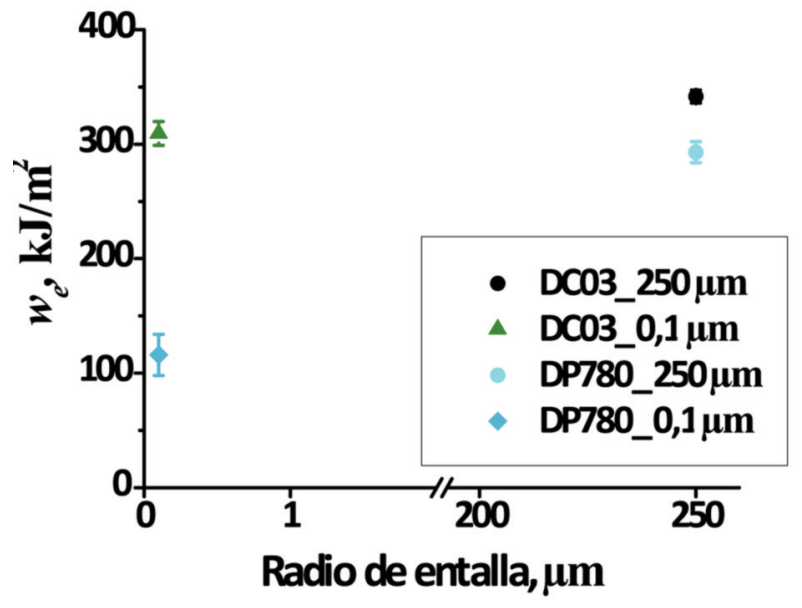

Figura 11. Evolución del $w_{e}$ en función del radio de entalla.

Figure 11. $w_{e}$ evolution depending on notch radii.

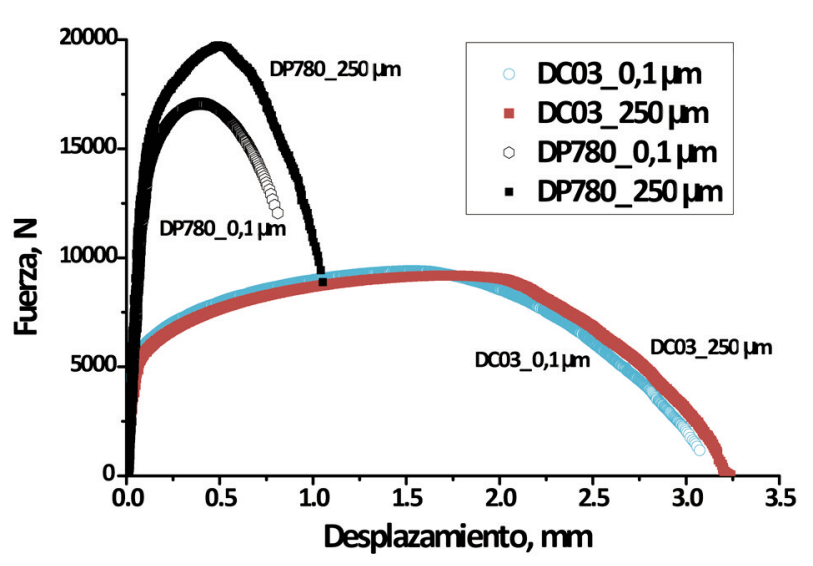

Figura 12. Curvas F vs. despl. para el ligamento de $15 \mathrm{~mm}$ de aceros DC03 y DP780.

Figure 12. F vs. despl. curves for ligament of $15 \mathrm{~mm}$ in DC03 and DP780 steels.

tura, se puede tomar que el parámetro de fractura intrínseco del material es el obtenido con la grieta más aguda. Por tanto, se debería considerar que en el método TEF el valor intrínseco de $w_{\mathrm{e}}$ es el calculado a partir de la propagación de grietas por fatiga. En el caso del acero DP780, las muestras con radios de entalla de $250 \mu \mathrm{m}$ sobreestiman claramente los valores de $w_{\mathrm{e}}$.

\section{CONCLUSIONES}

De acuerdo con los resultados experimentales obtenidos del trabajo esencial de fractura para aceros 
DP780 y DC03, se pueden extraer las siguientes conclusiones:

- Al igual que en aceros de bajo carbono, como el acero DC03 aquí estudiado, el método TEF puede ser utilizado en aceros AHSS, como por ejemplo los aceros de fase dual (Dual Phase) de $800 \mathrm{MPa}$ de resistencia mecánica y es válido para determinar la tenacidad de fractura de este tipo de aceros. Estos resultados abren la posibilidad de aplicar dicho método a otros aceros AHSS de mayor resistencia mecánica, dado que existe la metodología experimental necesaria para determinar si se cumplen las condiciones de plasticidad impuestas por el ensayo.

- Se observa una gran dependencia del valor de $w_{\mathrm{e}}$ con el radio de entalla en el acero DP780, mientras que el acero DC03 esta dependencia es mucho menor.

- La metodología TEF se postula como una buena herramienta de evaluación de la tenacidad de fractura para poder ser utilizada como parámetro mecánico en la caracterización a fractura de aceros AHSS.

\section{Agradecimientos}

Los autores agradecen el financiamiento recibido de ACC1Ó (contratos TECCTA10-1-0001 y TECCTA11-1-0006).

\section{REFERENCIAS}

[1] Recurso en línea: http://www.worldautosteel.org

[2] ASTM Standard E1820-01. Standard Test Method for Measurement of Fracture Toughness. ASTM International, West Conshohocken, Pennsylvania, USA, 2001.
[3] O. Akourri, M. Louah, A. Kifani, G. Gilbert y G. Pluvinage, Eng. Fract. Mech. 65 (2000) 491-505.

[4] K.B. Broberg, J. Mech. Phys. Solids 23 (1975) 215-237.

[5] B. Cotterell y J.K. Reddel, Int. J. Fracture 13 (1977) 267-277.

[6] Y. Marchal y F. Delannay, Int. J. Fracture 80 (1996) 295-310.

[7] Y. Marchal, K. Schmidt, T. Pardoen, R. Knockaert. I. Doghri y Y. Delannay, EMAS (ECF 11) Mechanisms and Mechanics of Damage and Failure, Francia, 1996, pp. 2.259-2.265.

[8] T. Pardoen, F. Hachez, B. Marchioni, P.H. Blyth y A.G. Atkins, J. Mech. Phys. Solids 52 (2004) 423-452.

[9] S.V. Kamat, J.P. Hirth y F.W. Zok, Acta Materialia 44 (1996) 1.831-1.838.

[10] E. Clutton y D.R. Moore, ESIS EWF Protocol: Essential work of fracture and cohesive zone fracture toughness. ESIS 2003.

[11] A.B. Martínez, J. Gámez-Pérez, M. Sánchez-Soto, J.I. Velasco, O.O. Santana y M.L. Maspoch, Eng. Fail. Anal. 16 (2009) 2.604-2.617.

[12] W. Jingshen y M. Yiu-Wing, Polym. Eng. Sci. 12 (1996) 2.275-2.288.

[13] J. Gamez-Perez, O. Santana, A.B. Martinez y M.Ll. Maspoch, Polym. Test. 27 (2008) 491-497.

[14] T. Pardoen, Y. Marchal y F. Delannay, Journal of Mechanics Solids 47 (1999) 2.093-2.123.

[15] G. Lacroix, T. Pardoen y P.J. Jacques, Acta Materialia 56 (2008) 3.900-3.913.

[16] D. Gutiérrez, A. Lara, D. Casellas y J.M. Prado, Advanced Materials Research 89-91 (2010) 214-219.

[17] R. Knockaert, I. Doghri, Y. Marchal, T. Pardoen y F. Delannay, Int. J. Fracture 81 (1996) 383-399.

[18] V.V. Chaudhari, D.M. Kulkarni y R. Prakash, Fatigue Fract. Eng. Mater. Struct. 32 (2009) 975-986. 